\title{
DISTRIBUIÇÃO DOS DIÂMETROS DOS TRONCOS DAS ESPÉCIES MAIS IMPORTANTES DO CERRADO NA ESTAÇÃO FLORESTAL DE EXPERIMENTAÇÃO DE PARAOPEBA (EFLEX)-MG'
}

\author{
Manoel Cláudio da Silva Júnior ${ }^{2}$ \\ Alexandre Francisco da Silva ${ }^{3}$
}

Recebido em 20-2-88. Aceito em 13-10-88.

\begin{abstract}
RESUMO - Este estudo foi realizado no cerrado (sensu stricto) da Estação Florestal de Experimentação de Paraopeba-MG, pertencente ao Instituto Brasileiro de Desenvolvimento Florestal (IBDF). Para o levantamento florístico utilizou-se o método de quadrantes, incluindo-se os indivíduos com diâmetro mínimo do tronco de $5 \mathrm{~cm}$ ao nível do solo. Foram alocados 25 pontos de amostragem em cada uma das 20 áreas selecionadas pela homogeneidade fisionômica. A distribuição dos diâmetros dos 2.000 indivíduos amostrados apresentou-se não balanceada, indicando uma grande representação $(66,55 \%)$ na menor classe de diâmetro $(5-10 \mathrm{~cm})$, podendo isto ser devido ao restabelecimento das populações na área após o desmatamento ocorrido em 1952. Esta hipótese sugere que 30 anos seria um prazo insuficiente para o estabelecimento da rotação de corte naquele cerrado. Levantaram-se a situação atual e as hipóteses quanto aos prováveis problemas enfrentados para o estabelecimento de algumas das populações que mais se destacaram pelo índice do valor de importância (IVI) e pelo número mínimo de indivíduos amostrados $(\mathrm{n}=50)$ : Qualea parviflora Mart., Byrsonima crassa Nied., Qualea grandiflora Mart., Erythroxylum suberosum St. Hill., Eugenia dysenterica D.C., Kielmeyera grandiflora (Wawra) Saddi, Dimorphandra mollis Benth., Curatella americana L., Terminalia argentea Mart., Annona crassiflora Mart., Machaerium opacum Vog. Concluiu-se que a atividade de extração de minhocuçus na Estação tem contribuído negativamente para o estabelecimento de novos indivíduos na área e, para a recuperação das populações, recomenda-se o plantio de mudas produzidas na própria EFLEX. Para o sucesso de qualquer medida com este objetivo é essencial uma efetiva fiscalização da área.
\end{abstract}

Palavras-chave: dinâmica populacional, cerrado, distribuiçào de diâmetros.

ABSTRACT - This study was carried out in 200ha of the Experimental Forest Station (EFLEX) of Paraopeba-MG with the purpose of evaluating the trunk diameter distribution of local "cer-

\footnotetext{
${ }^{1}$ Trabalho apresentado no 37\% Congresso Nacional de Botânica, Ouro Preto (MG), de 19 a 26 de janeiro de 1986

${ }^{2}$ Universidade de Brasília, Departamento de Engenharia Florestal - Caixa Postal 152807 - BrasíliaDF - CEP 70919

${ }^{3}$ Universidade Federal de Viçosa, Departamento de Biologia Vegetal - Viçosa-MG - CEP 36570
} 
rado" vegetation. Twenty sampling plots were randomly distributed through out the experimental area and the floristic composition was studied using the point-centered-quarter method, including individuals of at least $5 \mathrm{~cm}$ diameter at the ground level. Twenty-five sampling points were located in each plot. Diameter distribution of 2,000 individuals was unbalanced, with $66,55 \%$ of these in the smallest diameter class $(5-10 \mathrm{~cm})$. This could be due to population restablishment caused by deforestation that took place in 1952. This suggests that a 30 year rotation period might not be long enough for managing "cerrado" vegetation. The situation of populations with at least 50 individuals sampled or with highest importance value (IVI) was studied: Qualea parviflora Mart., Byrsonima crassa Nied. Qualea grandiflora Mart., Erythroxylum suberosum St. Hill., Eugenia dysenterica D.C., Kielmeyera grandiflora (Wawra) Saddi, Dimorphandra mollis Benth., Curatella americana L., Terminalia argentea Mart., Annona crassiflora Mart. and Machaerium opacum Vog. Hypothesis were postulated to explain problems that may affect the establishment of the species. Observations indicate that digging for earthworms has adversely affected the establishment of new individuals. It is recomended that the native seedlings being produced at EFLEX could be used to regenerate natural "cerrado"; however effective enforcement is needed to guarantee its success.

Key words: population dynamics, "cerrado", distribution of diameters.

\section{Introdução}

Nos sistemas temperados as idades das árvores podem ser avaliadas pelo número de anéis de crescimento (Daubenmire, 1968). Uma outra forma de avaliação é através da medição dos diâmetros dos troncos (Daubenmire, 1968; Harper, 1977), quando se espera que o tamanho da circunferência do tronco reflita a idade do indivíduo. Este procedimento pode ser utilizado nos sistemas tropicais, nos quais os anéis de crescimento não são biem delimitados.

Estudando 21 espécies florestais na Amazônia, Moraes (1970) chegou a resultados indicativos de que, quando em um determinado período havia acréscimo na velocidade de crescimento da circunferência do tronco, isto se verificava, de um modo geral, em todos os indivíduos de uma mesma espécie.

Mesmo que existam problemas quanto à avaliação da idade através da medição dos diâmetros das árvores, esta fornece a estrutura de tamanho de uma população, que é de grande importância para estudos florestais concernentes a predições sobre a produção daqueles recursos naturais. Neste sentido, a idade real das árvores não é um parâmetro muito importante (Harper, 1977).

Considerando uma comunidade em equilíbrio, Daubenmire (1968) ressalta que deveria haver uma grande produção de sementes, seguida de germinação satisfatória e, inicialmente, uma alta taxa de mortalidade, que seria decrescente nas idades mais avançadas até que se completasse uma seqüência em que as árvores estariam em idade de produção de sementes. Neste caso, esperar-se-ia uma série completa de classes de idade para cada uma de suas espécies.

Uma comunidade com esta distribuição de classes de diâmetros ou idade poderia ser considerada clímax, pois apresentaria um domínio permanente do habitat. Por outro lado, se as classes de diâmetros se apresentassem interrompidas ou truncadas, o ciclo de vida da espécie considerada não estaria completando-se, o 
que indicaria um desequilíbrio ocasionado por fatores locais, uma vez que a estrutura da população é sensível às condições do ambiente (Daubenmire, 1968).

Os histogramas de freqüências nas classes de diâmetros podem retratar os acontecimentos por que passou uma determinada floresta. Assim, podem-se supor as perturbações ocorridas como: ataque de insetos, doenças, abate seletivo, não estabelecimento de plântulas, baixo índice de germinação etc.

O presente trabalho tem como objetivo contribuir para o conhecimento da dinâmica populacional da vegetação arbórea de uma área de cerrado, através da distribuição dos diâmetros dos troncos das espécies mais representativas, a fim de sugerir estratégias que possam ser tomadas para levar aquela comunidade aos menores níveis de perturbação possíveis.

\section{Material e métodos}

A Estação Florestal de Experimentação de Paraopeba-MG (EFLEX) está situada a $19^{\circ} 20^{\prime} \mathrm{S}$ e $44^{\circ} 20^{\prime} \mathrm{W}$, em altitude que varia dos 734 aos $750 \mathrm{~m}$. A área total da Estação é de aproximadamente 200ha, estando totalmente cercada e subdividida em 59 talhões delimitados por aceiros. Destes talhões, 45 são cobertos por vegetação de regeneração natural de diferentes fisionomias e os restantes estão destinados à experimentação e outros usos.

O clima foi caracterizado, segundo os procedimentos recomendados por Thornthwaite \& Mather (1957), como subtropical úmido, com verão chuvoso e estação seca que vai de abril a setembro (Fig. 1).

Para o estudo da vegetação empregou-se o método de quadrantes (Cottam \& Curtis, 1956), escolhendo-se 17 talhões com vegetação de cerrado (sensu stricto) fisionomicamente homogênea, nos quais foram selecionadas 20 áreas de estudo. Em cada área foram alocados 25 pontos. Todos os indivíduos que possuíam diâmetro igual ou superior a $5 \mathrm{~cm}$ ao nível do solo foram amostrados.

Foram selecionadas algumas das populações que alcançaram as primeiras posições em função do IVI, calculado segundo Martins (1979), e em função do número de indivíduos amostrados, que foi estipulado em 50 como o mínimo. Estes foram dispostos em classes de diâmetros com amplitude de $5 \mathrm{~cm}$, na forma de histogramas, conforme procedimento proposto por Spiegel (1976).

Para a avaliação da distribuição dos diâmetros foi calculado o quociente " q", proposto por Liocourt (1898) apud Meyer (1952), segundo o qual, numa população com distribuição balanceada dos diâmetros, a redução do número de indivíduos de uma classe para a seguinte deveria ocorrer numa razão constante (quociente “"q"). Por outro lado, em florestas com distribuição não balanceada dos diâmetros, esta redução seria diretamente proporcional ao aumento dos diâmetros (Leak, 1964). Assim, este quociente foi obtido pela divisão do número de indivíduos de uma classe pelo número de indivíduos da classe seguinte. 


\section{Resultados e discussão}

Distribuição das freqüências nas classes de diâmetros

A Fig. 2 mostra que a distribuição de freqüências das classes de diâmetros para os 2.000 indivíduos não foi balanceada, sendo os valores "q" iguais a 2,88 , $3,25,3,88,3,5,2,0$ e 2,0 .

$\mathrm{O}$ maior número de indivíduos nas classes inferiores de diâmetros pode indicar que a maioria das populações está em fase inicial de estabelecimento. Por outro lado, em se tratando da vegetação de cerrado, deve-se considerar que algumas das espécies apresentam porte menor, por ser esta a sua potencialidade genética.

A ausência de representação em duas das maiores classes pode significar que o ciclo de vida das espécies que alcançariam diâmetros maiores não está completando-se. Apesar da possibilidade do abate seletivo dos indivíduos maiores, é provável que os incêndios ocorridos em 1960 e 1968 tenham eliminado da comunidade os indivíduos.que ocupariam estas classes. Entretanto, considerandose também um desmatamento total da área em 1952, presume-se que a maioria das populações não tenha tido tempo suficiente para alcançar diâmetros maiores, ou seja, as populações estariam, ainda, em fase de restabelecimento. Esta hipótese, aliada à constatação de que $66,55 \%$ dos indivíduos amostrados pertencem à classe de $4-10 \mathrm{~cm}$ de diâmetro, sugere que para o cerrado 30 anos seria um prazo insuficiente para o estabelecimento da rotação de corte.

A Fig. 3 mostra a distribuição de freqüência das classes de diâmetros da população de Qualea parviflora Mart., que contou com 249 indivíduos amostrados.

Os quocientes “"q" apresentaram valores de 2,18, 2,16 e 6,20, que indicam uma redução quase constante do número de indivíduos nas primeiras classes, o que significaria uma distribuição balanceada. Entretanto, a redução da terceira para a quarta classe foi muito acentuada, indicando um menor número de indivíduos de maior porte do que era de se esperar para esta população.

Segundo Heiseke (1976), a madeira de Qualea parviflora é muito utilizada para tábuas, postes e mobílias, o que indica a possibilidade de ocorrência de um abate seletivo, para a utilização da madeira produzida por indivíduos maiores.

Esta espécie apresentou a maior densidade e área basal por hectare entre todas as outras amostradas (Silva Júnior, 1984). Estudando as tipologias florestais do cerrado na região central de Minas Gerais, Heiseke (1976) afirmou que $Q$. parviflora estava representada em todos os tipos de cerrado, com alto grau de abundância, dominância e volume relativo, observando, ainda, o grande poder de competição e o potencial de crescimento da espécie.

A Fig. 4 representa a distribuição dos diâmetros da população de Byrsonima crassa Nied., com os 147 indivíduos amostrados distribuídos em quatro classes de diâmetros. Observa-se que a maioria dos indivíduos (130) coloca-se na classe de $5-10 \mathrm{~cm}$ e que as classes seguintes apresentam número de indivíduos bastante reduzido. 
Os quocientes “"q" de 9,29, 7,0 e 2,0 indicam uma distribuição não balanceada, devido à concentração de indivíduos na classe inferior. A hipótese de que a população esteja em fase inicial de restabelecimento na área parece pouco provável, uma vez que a grande maioria dos indivíduos floresceu e frutificou durante a realização dos trabalhos de campo, mostrando sua maturidade fisiológica. Supõese, desta forma, que estes indivíduos sejam provenientes de reprodução vegetativa, o que lhes proporcionou, mesmo com pequeno porte, a maturidade sexual ou, em outra hipótese, a possibilidade de um abate excessivo dos indivíduos de maior porte. Segundo os mateiros da região, esta espécie fornece excelente madeira para lenha de fogão, o que reforça o raciocínio anterior.

Em estudo da distribuição dos diâmetros em uma faixa de cerrado na Fazenda Água Limpa (FAL), no Distrito Federal, Felfili \& Silva Júnior (1986) constataram para $B$. crassa uma distribuição com a maioria dos indivíduos na menor classe $(6-10 \mathrm{~cm})$. Ao contrário de Paraopeba, o cerrado da FAL não sofreu qualquer desmatamento em grande escala; apenas os incêndios têm sido mais freqüentes.

A população de Quatea grandiflora Mart. contou com 116 indivíduos distribuídos em cinco classes sucessivas (Fig. 5). Os quocientes " $q$ " de 2,16, 3,20, 3,33 e 1,5 indicam que a distribuição não foi balanceada, embora a curva apresente, em seu aspecto geral, a forma de um "J" invertido, previsível para distribuições balanceadas. Os valores crescentes dos quocientes indicam que o número de indivíduos encontrados nas sucessivas classes foi menor que o esperado. A redução mais acentuada, a partir da segunda até a quarta classe, indica que provavelmente os indivíduos com maiores diâmetros foram abatidos, pois, segundo Heiseke (1976), a madeira desta espécie, assim como a de $Q$. parviflora, também é procurada para a utilização em forma de tábuas, postes e mobílias.

$\mathrm{O}$ fato de a espécie ter florescido e frutificado abundantemente na área, juntamente com o grande número de indivíduos de menor porte, sugere o seu grande potencial para a exploração de ambiente.

A população de Erythroxylum suberosum St. Hill. contou com 140 indivíduos distribuídos em três classes de diâmetros (Fig. 6). A grande maioria dos indivíduos (117) concentrou-se na menor classe de diâmetro $(5-10 \mathrm{~cm})$. Os quocientes “"q" foram de 5,6 e 10,5.

Esta espécie obteve o terceiro valor para a densidade e o sétimo valor para a área basal, evidenciando o grande número de indivíduos de pequeno porte. Estes floresceram e frutificaram na área, o que sugere sua maturidade fisiológica. $\mathrm{O}$ comportamento da população de $E$. suberosum é bastante semelhante ao da população de $B$. crassa, sendo assim levantadas hipóteses semelhantes àquelas sugeridas anteriormente, como um pequeno porte potencial e possibilidade de indivíduos provenientes da reprodução vegetativa.

Os 110 indivíduos de Eugenia dysenterica D.C. distribuíram-se em quatro classes de diâmetros (Fig. 7).

Os quocientes " $q$ " de 1,79, 3,09 e 2,75 mostram que a redução do número de indivíduos foi mais acentuada da segunda para a terceira classe do que desta 
para a quarta. Seria necessário, então, um maior número nas classes inferiores para que a população apresentasse uma distribuição balanceada.

Esta espécie produz frutos saborosos que são muito procurados pelo homem, o que de certa forma reduz as chances de corte dos indivíduos de maior porte para outra utilização. Este fato poderia acarretar um menor número de sementes para o estoque do solo, devido à retirada constante dos frutos, o que levaria a uma redução do número de indivíduos de menor porte.

Quanto à regeneração de $E$. dysenterica, Rizzini (1979) constatou em observações realizadas em 1967, na Estação, grande número de novos indivíduos estabelecidos por sementes. Afirma ainda o autor que o processo de germinação exige uma seqüência bem determinada de condições ecológicas e que qualquer distúrbio na área pode limitar a regeneração por sementes. Neste caso, a atividade dos chamados "minhoqueiros" certamente está interferindo no estabelecimento de novos indivíduos.

A Fig. 8 apresenta a distribuição de freqüências nas classes de diâmetros para os 101 indivíduos da população de Kielmeyera grandiflora (Wawra) Saddi, que se distribuíram em três classes de diâmetros. Os quocientes " "q" de 11,5 e 8,0 indicam a grande representação nas menores classes, com 92 indivíduos.

Segundo informações do diretor da Estação, esta espécie, embora não apresente problemas quanto à germinação, cresce muito lentamente, podendo daí suporse que não houve tempo suficiente, após o desmatamento de 1952, para que os indivíduos dessa população atingissem diâmetros maiores.

A Fig. 9 apresenta a ditribuição dos diâmetros dos 72 indivíduos amostrados de Dimorphandra mollis Benth., que ficaram distribuídos em três classes de diâmetros.

Os quocientes “"q" de 1,73 e 1,83 indicam uma distribuição próxima daquela que se considera balanceada. A espécie floresceu e frutificou abundantemente durante os trabalhos de campo, mostrando não haver problemas quanto à polinização. Foram observadas muitas plantas pequenas, o que indica que o ciclo de vida está-se completando. Segundo Rizzini (1979), em observações feitas em Paraopeba, foram encontradas plantas jovens da espécie, estabelecidas através de sementes, como também pela brotação de raízes gemíferas, sugerindo daí que ela possui um grande potencial para a exploração do ambiente local.

A Fig. 10 apresenta a distribuição para a população de Curatella americana L. Foram amostrados 46 indivíduos, que se distribuíram em seis classes de diâmetros. Os quocientes " $q$ " de 0,6, 1,25, 2,0, 2,0 e 3,0 indicam, considerando-se a curva a partir da segunda classe, que parece haver um pequeno número de indivíduos nas classes superiores. O mais provável é que estes tenham sido abatidos. Observa-se, ainda, que o número de indivíduos na classe de $5-10 \mathrm{~cm}$ foi menor que o esperado, o que sugere que o ciclo de vida da espécie não está completando-se. Neste caso, à medida que as árvores das classes superiores morrerem, não deverá haver efetivo de indivíduos da classe inferior para substituí-las, o que certamente provocará uma redução acentuada na densidade de C. americana na Estação. Su- 
põe-se que o menor número de indivíduos de pequeno porte da espécie seja devido a fatores como a redução da polinização, ou a problemas na germinação, de predação excessiva de sementes e/ou plântulas ou mesmo pela atividade dos "minhoqueiros".

Segundo Laboriau et al. (1964), foram encontradas, em diferentes localidades de Minas Gerais, plântulas de C. americana com restos reconhecíveis de sementes, bem como plantas jovens com sistema radicular autônomo, o que indica regeneração via sementes. Além disso, Rizzini (1979) constatou que esta espécie apresenta plântulas muito frágeis e de crescimento muito lento, devendo, por isso, exigir condições particularmente favoráveis para estabelecerem-se. Rizzini (1971) constatou que as sementes de $C$. americana apresentavam-se de 23 a $80 \%$ brocadas, o que indica uma alta predação, podendo ser este um outro problema enfrentado pela espécie.

A população de Terminalia argentea Mart. contou com 56 indivíduos amostrados, que se distribuíram em seis classes de diâmetros (Fig. 11). Observou-se a ausência de representação em quatro classes consecutivas, o que sugere uma interferência prolongada de algum fator ou que a população está-se restabelecendo na área. Assim, os indivíduos maiores, provavelmente, ainda não alcançaram seus diâmetros máximos, enquanto o único indivíduo da última classe seria remanescente do desmatamento de 1952.

Os quocientes “"q" foram de 2,57, 4,67, 3,0 e 1,0 mostrando uma distribuição não balanceada. A madeira de T. argentea é muito procurada, a nível regional, para o fabrico de postes e mobílias (Heiseke, 1976), o que poderia justificar a queda acentuada do número de indivíduos nas classes intermediárias.

A Fig. 12 mostra o comportamento da população de Annona crassiflora Mart., com seus 50 indivíduos distribuídos em cinco classes de diâmetros. Os quocientes " q" foram de $1,5,1,75,1,33$ e 6,0 . Como se observa, as primeiras classes apresentam-se com uma tendência ao balanceamento, acentuando-se, entretanto, a redução dos indivíduos da quarta para a quinta classe.

Esta espécie, tal como a Eugenia dysenterica, produz grandes frutos, conhecidos como araticum, que são constantemente retirados pelo homem, sendo, assim, pouco provável que as grandes árvores tenham sido abatidas para outra finalidade. Supõe-se, desta forma, que a população de $A$. crassiflora esteja em fase avançada de restabelecimento na área.

Para a população de Machaerium opacum Vog., foram amostrados 56 indivíduos, que se distribuíram em quatro classes de diâmetros (Fig. 13). Os quocientes “" $q$ " foram calculados em 1,4, 2,86 e 7,0, indicando uma distribuição não balanceada.

Constatou-se uma redução muito acentuada do número de indivíduos da terceira para a quarta classe, sugerindo um abate para o aproveitamento das árvores de maiores dimensões.

Como ficou constatado, as populações que apresentaram um pequeno número de indivíduos nas classes inferiores de diâmetros muito provavelmente sofreram 
influência das atividades dos "minhoqueiros", que, utilizando enxadões, revolvem as primeiras camadas do solo em busca dos minhocuçus, cortando, ao mesmo tempo, as plântulas e indivíduos jovens, e impedindo, com esta prática, a regeneração natural da vegetação.

\section{Conclusões}

Apesar de a EFLEX-Paraopeba estar totalmente cercada, o homem como agente perturbador do ambiente é um fato que se constatou, inúmeras vezes, durante a realização deste trabalho, principalmente a ação dos "minhoqueiros". Esta afirmativa é provada, já que, de maneira geral, a distribuição dos diâmetros sugeriu algum tipo de perturbação que, provavelmente, foi introduzida pela ação humana. A importância dos dados apresentados processa-se mais a nível de constatação da condição atual das populações estudadas do que para o reconhecimento dos vários fatores que pudessem causar os problemas sugeridos, para o que seriam necessários estudos mais detalhados e duradouros.

Para o restabelecimento adequado das populações estudadas e para a vegetação em geral, aconselhar-se-ia o plantio das espécies nativas da área, utilizando-se as mudas que já são produzidas na própria EFLEX-Paraopeba.

Diante do desmatamento acelerado e incontrolável que se tem observado na região de Paraopeba, onde a vegetação é transformada em carvão para alimentar as indústrias locais, a EFLEX-Paraopeba muito em breve, certamente, será a única área de preservação da região, constituindo-se em valioso "banco gênico" e o único representante do que teria sido a flora local. Para tanto, faz-se necessário que o desenvolvimento daquela comunidade prossiga com os menores distúrbios possíveis, o que só poderá acontecer caso o IBDF exerça uma fiscalização severa, através do aumento efetivo da guarda florestal, a fim de coibir energicamente a penetração dos "minhoqueiros" e quaisquer outros, cujas atividades possam atentar contra o patrimônio biológico daquela Estação.

\section{Referências Bibliográficas}

COTTAM, G. \& CURTIS, J.T. 1956. The use of distance measurements in phytosociological sampling. Ecology 37(3): 451-460.

DAUBENMIRE, R. 1968. Plant communities: a textbook of plant synecology. New York, Harper \& Row Publ.

FELFILI, J.M. \& SILVA JÚNIOR, M.C. 1986. Distribuição dos diâmetros numa faixa de cerrado na Fazenda Água Limpa (FAL)-DF. In: VI Congresso da Sociedade Botânica de São Paulo. Campinas, 1986. Resumos p. 85

HARPER, J.L. 1977. Population biology of plants. London, Academic Press.

HEISEKE, D.R. 1976. Estudos de tipologias florestais do cerrado na região central de Minas Gerais. PNUD/FAO/IBDF/BRA-45. Série Técnica. Brasilia.

LABORIAU, L.G.; VALIO, I.F.M. \& HERINGER, E.P. 1964. Sobre o sistema reprodutivo das plantas dos cerrados I. An. Acad. Bras. Ciênc. 36(4): 449-464. 
LEAK, W.B. 1964. An expression of diameter distribution for unbalanced, uneven-aged stands and forests. Forest Science 10: 39-51.

MARTINS, F.R. 1979. O método de quadrantes e a fitossociologia de uma floresta residual do interior do Estado de São Paulo: Parque Estadual de Vassununga. Tese de Doutoramento. Universidade de São Paulo, São Paulo.

MEYER, H.A. 1952. Structure, growth and drain in balanced uneven-aged forests. Journal of Forest 50: 85-92.

MORAES, V.H.F. 1970. Periodicidade de crescimento do tronco de árvores da floresta amazônica. Pesquisa Agropecuária Brasileira 5: 315-320.

RIZZINI, C.T. 1971. Sobre as principais unidades de dispersão do cerrado. In: FERRI, M.G. coord. Simpósio sobre o cerrado 3. pp. 117-132. São Paulo, Edgard Blücher.

RIZZINI, C.T. 1979. Tratado de fitogeografia do Brasil: aspectos ecológicos e florísticos v. 2. HUCITEC, São Paulo.

SILVA JÚNIOR, M.C. 1984. Composição florística e parâmetros fitossociológicos do cerrado e sua relação com o solo na Estação Florestal de Experimentação de Paraopeba, MG. Dissertação de Mestrado. Universidade Federal de Viçosa, Viçosa, MG.

SPIEGEL, M.R. 1976. Estatística. São Paulo, McGraw-Hill.

THIBAU, C.E.; HEISEKE, D.H.; MOURA, P.V.; LAMAS, J.M. \& CESAR, R.L. 1975. Inventário preliminar expedito da Estação Florestal de Experimentação de Paraopeba em Minas Gerais. Brasil Florestal 6(21): 34-71.

THORNTHWAITE, C.W. \& MATHER, J.R. 1957. Instructions and tables for computing potential evapotranspiration and water balance. Publ. Climat. 10(3). Centreton, USA. 
LATITUDE : $199201 \mathrm{~S}$

LONGITUDE: $442201 \mathrm{~W}$

ALTITUDE : 734 m

TEMPERATURA MÉDIA ANUAL..........20,6 0

TEMPERATURA MÊS MAIS FRIO .........17,4 C

TEMPERATURA MÊS MAIS QUENTE........22,6 C

PRECIPITAÇÃO MÉOIA ANUAL...........1236 nm

EVAPOTRANSPIRAÇÃO POTENCIAL.......9967 m

DÉFICIT HÍDRICO............... $93 \mathrm{~mm}$

\section{PRECIPITAÇÃO EM mm}

----- EVAPOTRANSPIRAÇÃO POIENCIALL EM mm

EVAPOTRANSPIRAÇÃO REAL EM =m

EXCEDENTE DE ÁGUA

REPOSIÇÃO DE ÁGUA

V/D CONSUMO de ÁGUA

OÉFICIT HÍORICO

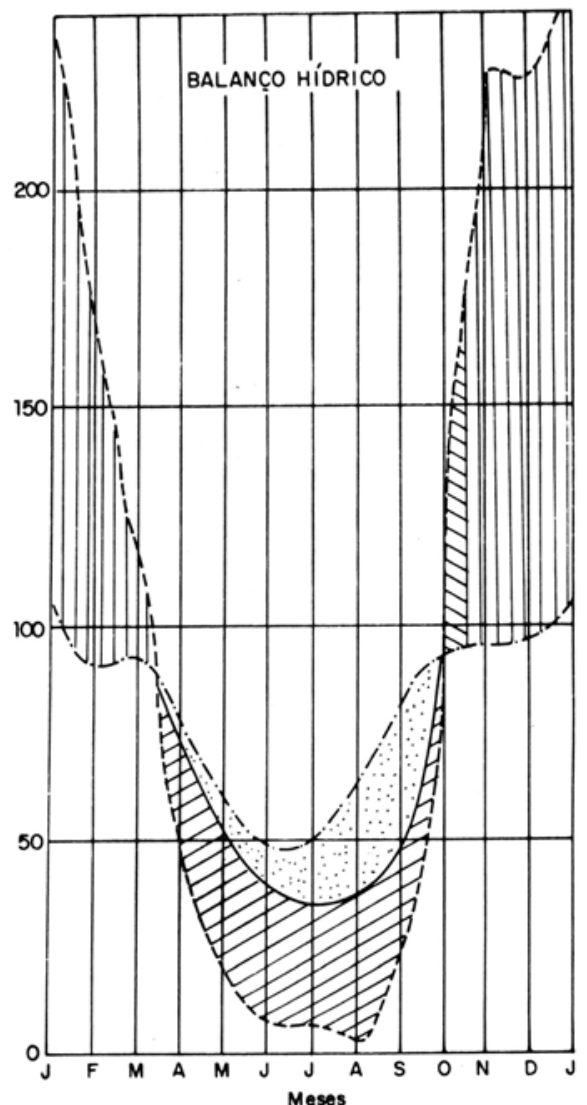

Meses

Figura 1 - Balanço hídrico climático representativo de Paraopeba (MG). Dados climáticos da própria EFLEX. (1959-1962) (THIBAU et al., 1975). 


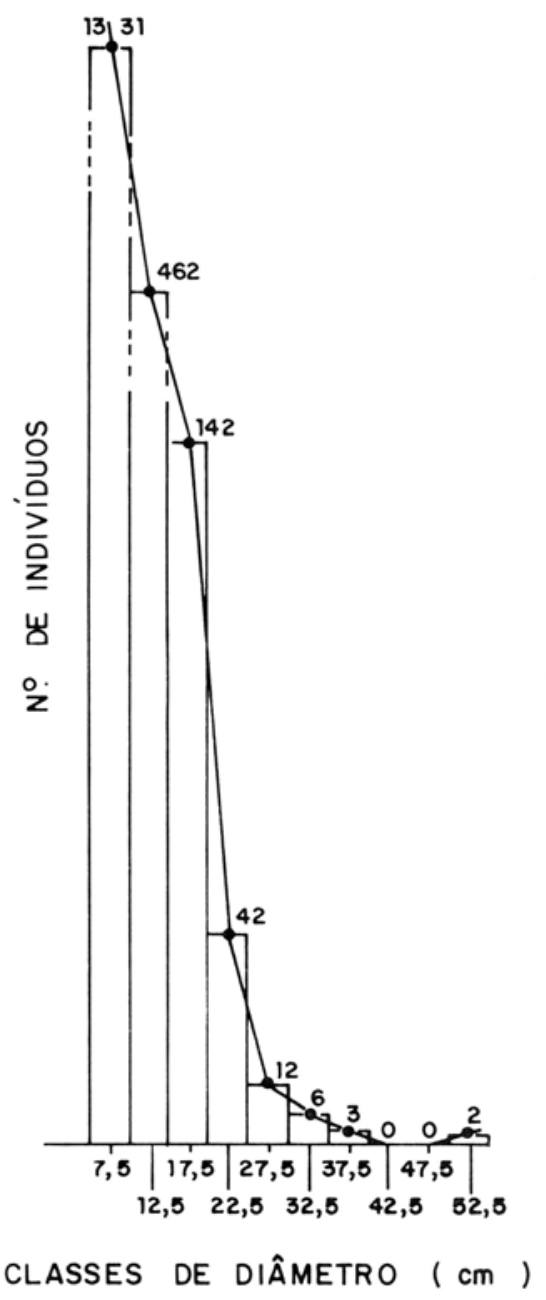

Figura 2 - Distribuição de freqüência nas classes de diâmetro para todos os indivíduos amostrados. 


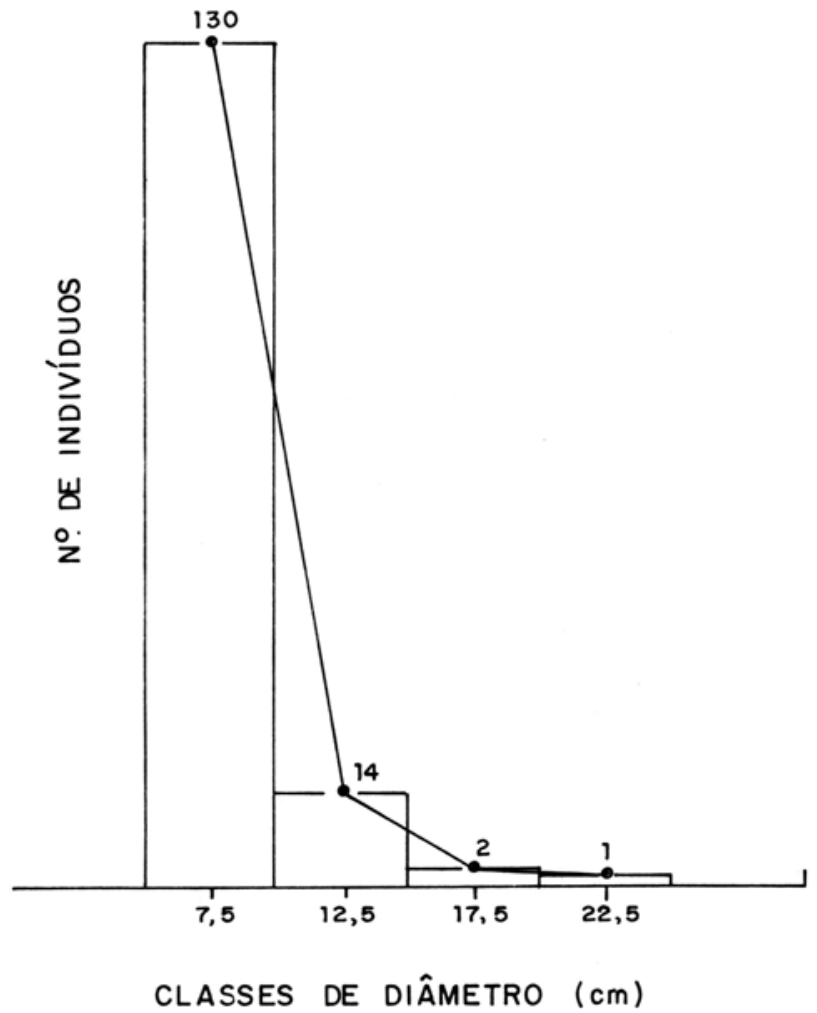

Figura 3 - Distribuição de freqüência nas classes de diâmetro para os indivíduos de Qualea parviflora Mart. 


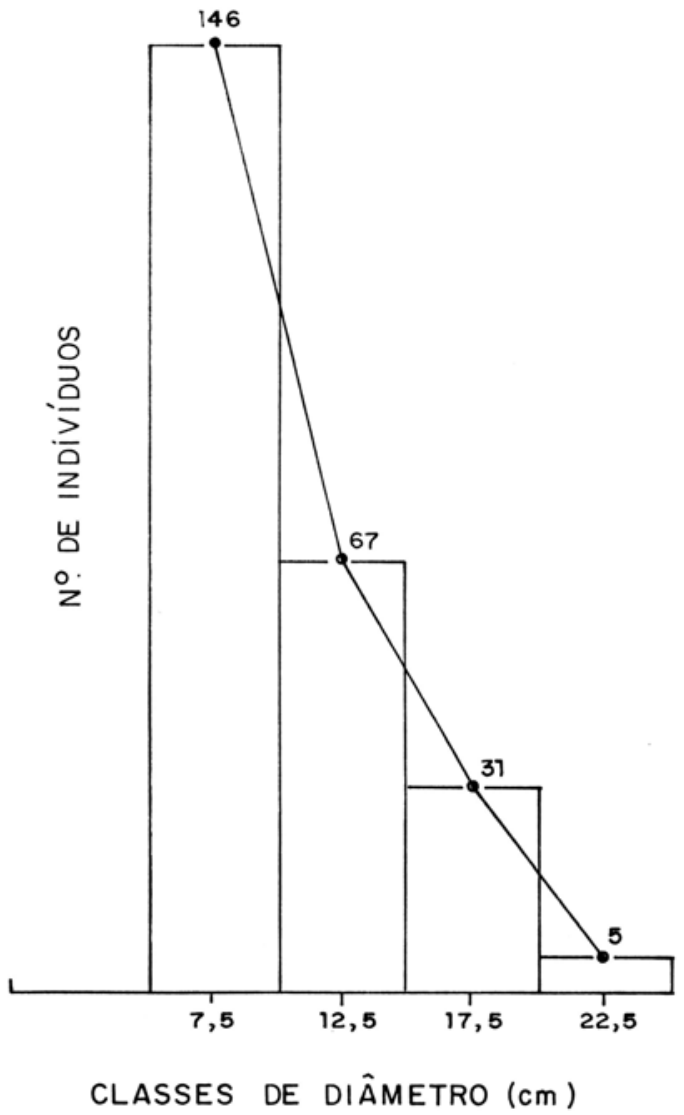

Figura 4 - Distribuição de freqüência nas classes de diâmetro para os indivíduos de Byrsonima crassa Nied. 


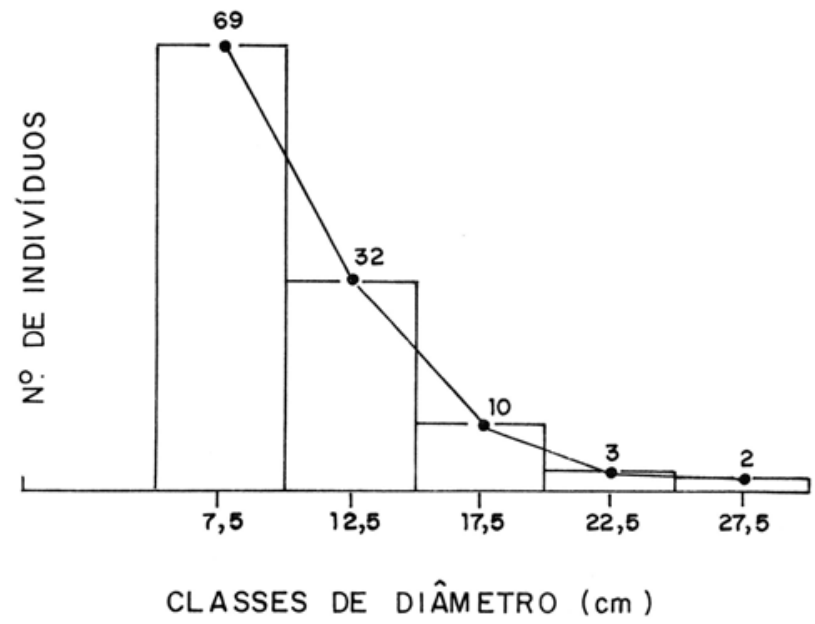

Figura 5 - Distribuição de freqüência nas classes de diâmetro para os indivíduos de Qualea grandiflora Mart. 


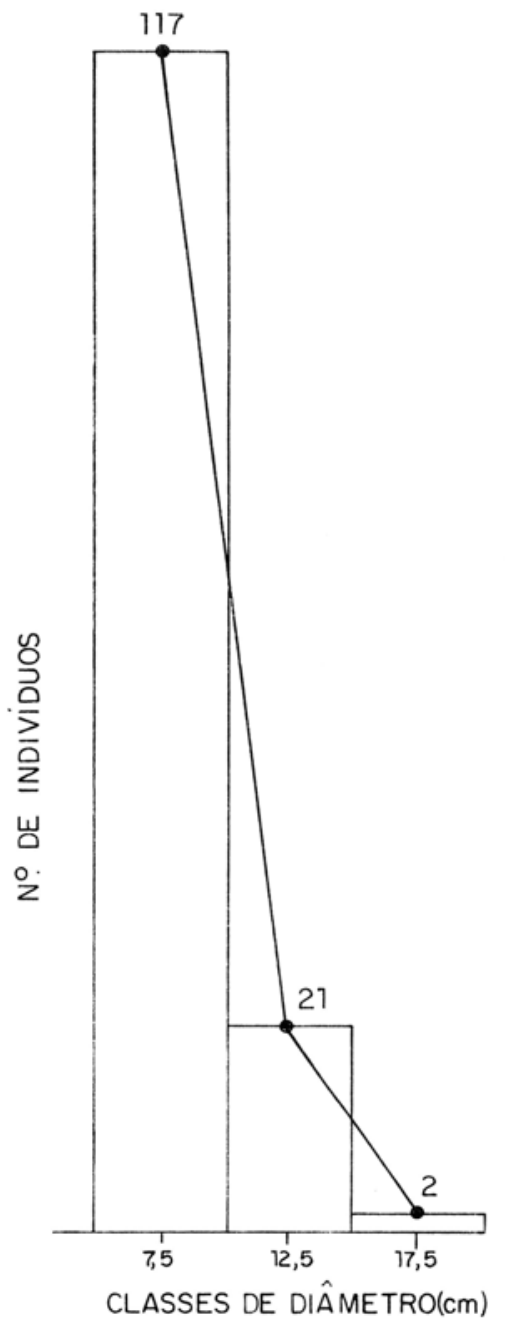

Figura 6-Distribuição de freqüência nas classes de diâmetro para os indivíduos de Erythroxylum suberosum St. Hill. 


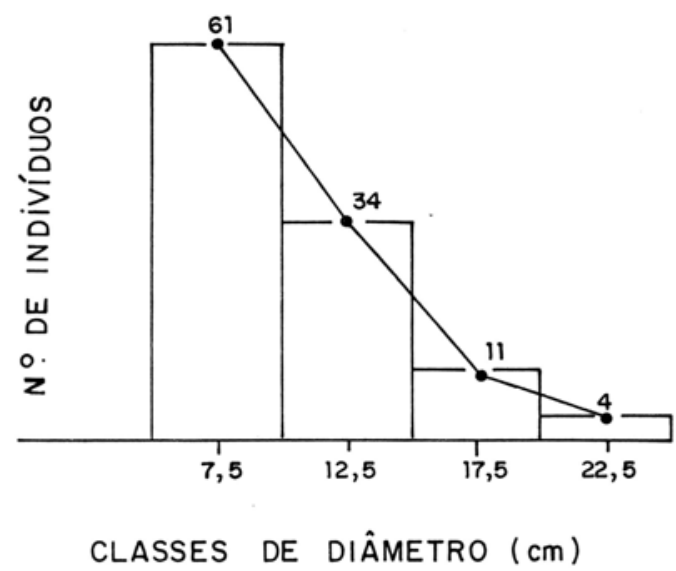

Figura 7 - Distribuição de freqüência nas classes de diâmetro para os indivíduos de $E u$ genia dysenterica D.C. 


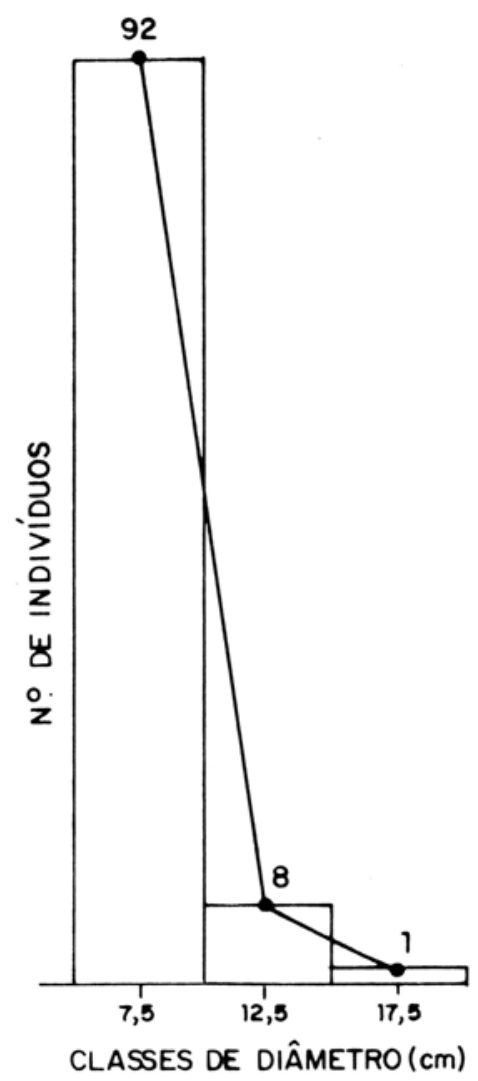

Figura 8 - Distribuição de freqüência nas classes de diâmetro para os indivíduos de Kielmeyera grandiflora (Wawra) Saddi. 


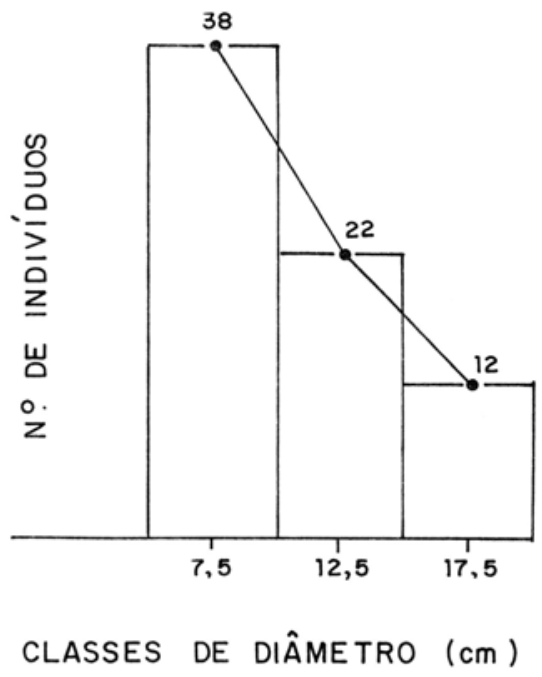

Figura 9 - Distribuição de freqüência nas classes de diâmetro para os indivíduos de $D i$ morphandra mollis Benth. 


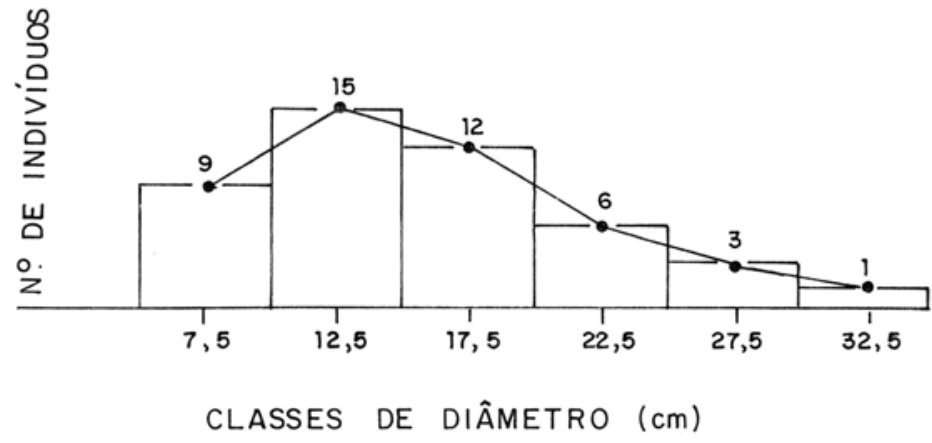

Figura 10 - Distribuição de freqüência nas classes de diâmetro para os indivíduos de $\mathrm{Cu}$ ratella americana $\mathrm{L}$.

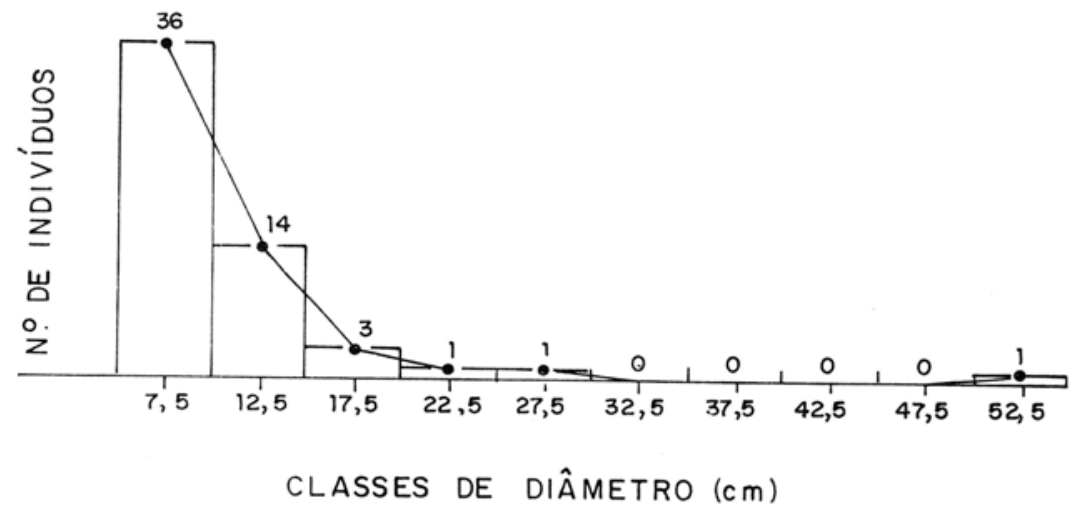

Figura 11 - Distribuição de freqüência nas classes de diâmetro para os indivíduos de Terminalia argentea Mart. 


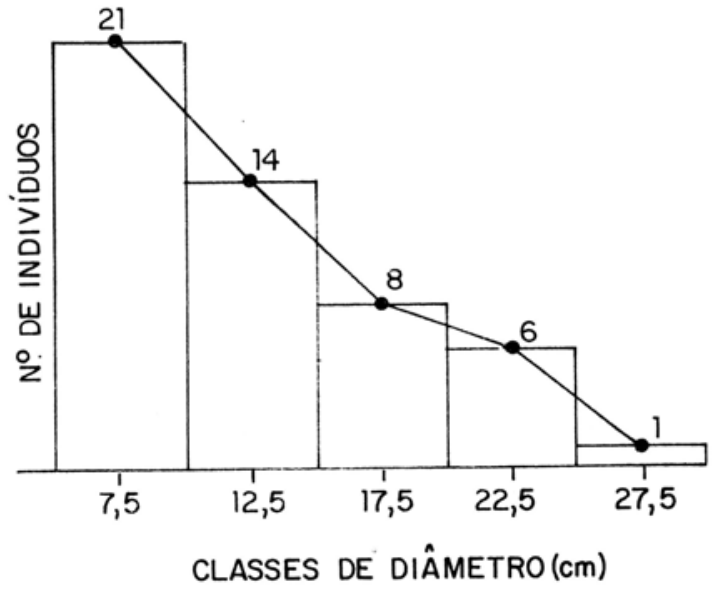

Figura 12 - Distribuição de freqüência nas classes de diâmetro para os indivíduos de $A n$ nona crassiflora Mart.

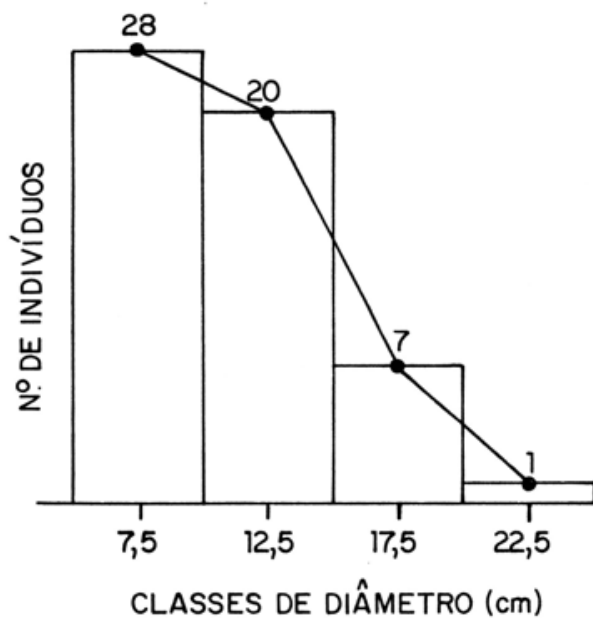

Figura 13 - Distribuição de freqüência nas classes de diâmetro para os indivíduos de $M a$ chaerium opacum Vog. 